\title{
Une copie de la chronique de Georges le Moine conservée à la bibliothèque de Patmos.
}

Le Patmiacus 7 (Sakellion) contient une partie considérable des livres III et IV de la chronique de Georges le Moine. C'est un manuscrit de beau parchemin, relié, haut de $18 \frac{1}{2}$ centimètres et large de 13; les quaternions sont de huit feuilles et numérotés. Au moment de la reliure, un bon nombre de feuillets avaient disparu au commencement, à la fin, et çà et là dans l'intérieur du volume. Les feuillets conservés sont au nombre de 295 . La page comprend 27 lignes avec une moyenne de 26 lettres par ligne.

L'écriture est toute de la même main, très soignée et régulière; elle paraît dater du XI ${ }^{2}$ e siècle. Le copiste n'a employé que fort peu d'abréviations; l'iota n'est jamais souscrit; on le trouve quelquefois ascrit. Les fautes d'orthographe sont très nombreuses.

Le Patmiacus ne donne pas les sommaires, ni de divisions en chapitres numérotés. Quelquefois on voit figurer dans la marge, en face d'un alinéa, l'indication du sujet, écrite de la première main. Le plus souvent cette indication donne avec la formule $\dot{\alpha} \varrho \chi \grave{\eta} \tau \tilde{\eta} S \beta \alpha \sigma \iota \lambda \varepsilon i \alpha_{S}$ le nom de l'enpereur dont le règne va être raconté.

La première feuille $d u$ manuscrit commence avec les mots [ $\pi \lambda o v] \sigma i \omega_{S}$

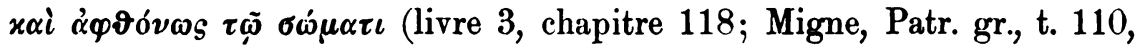
col. 396, dernière ligne).

Nous ne pouvons songer à reproduire ici la collation que nous possédons au complet: il faudra bien se contenter d'une description sommaire. Afin de lui donner toutes les chances d'être utile, nous nous arrêterons de préférence aux passages pour lesquels l'apparat critique de Muralt et les quelques travaux parus depuis, fournissent les renseignements les plus nombreux. A tous les extraits du Patmiacus que nous citerons, nous joindrons les variantes du Coislinianus 134, parfois celles du Coisl. 305, variantes que nous avons notées nous-mêmes à la Bibliothèque nationale. Malheureusement il nous a été impossible de faire le même travail pour l'important Coisl. 310, actuellement prêté au dehors. 


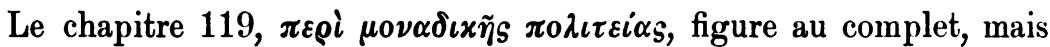
sans le titre, dans le Patmiacus $(\Pi)$. Il présente un bon nombre de variantes qui souvent le mettent d'accord avec Cedrenus. Pour les premières pages nous nous contenterons de signaler, avec les lacunes, quelques leçons intéressantes:

397, 43 (Migne) $\dot{\alpha} \tau \varepsilon \lambda \dot{\eta} s] \dot{\varepsilon} \nu \tau \varepsilon \lambda \dot{\eta} s$.

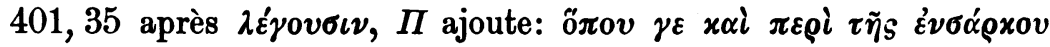

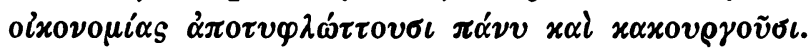

401, $51 \lambda \dot{v} \sigma \varepsilon \omega s] \lambda v \sigma \sigma \eta s$.

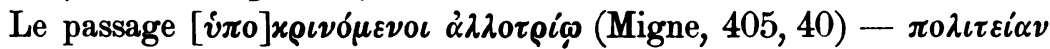
oï $\iota \nu \varepsilon_{S} \dot{\varepsilon} \nu(408,23)$ a disparu par suite de la perte d'une feuille.

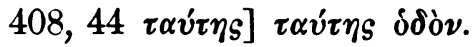

409, $26 \tau \varepsilon \lambda \varepsilon\left\llcorner 0 \tau \alpha^{\prime} \tau \eta \nu\right] \tau \varepsilon \lambda \varepsilon i o ́ \tau \eta \tau \alpha$.

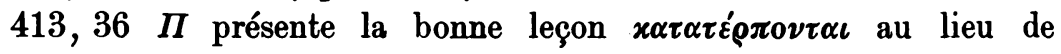

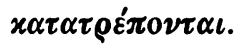

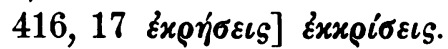

Un peu plus loin, il y a encore un feuillet perdu (

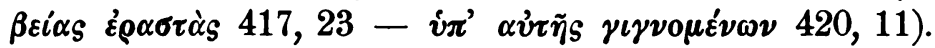

$\Pi$ omet les $\$ \$ 21-24$ de la fin du chapitre 119; aux chapitres 120 et 121, on le voit déjà se séparer du Mosquensis que Muralt a suivi, pour se ranger dans le groupe des Coisliniani 310 et 134 qui reproduisent, comme l'ont montré $M M$. Hirsch et de Boor ${ }^{1}$ ), la rédaction première de la chronique. Immédiatement après les mots $M \varepsilon \tau \dot{\alpha} \delta \grave{\varepsilon}$

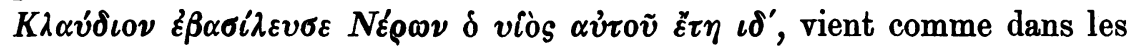
Coisliniani, l'histoire de Simon le magicien. Nous citerons ici les premières lignes de la version du Patmiacus, avec les variantes des Coisl. 134 et 305, et celles du Mosquensis:

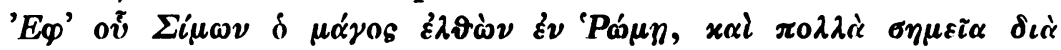


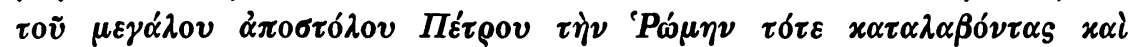

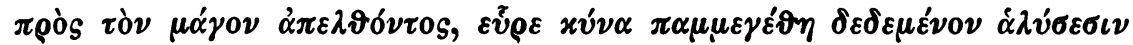

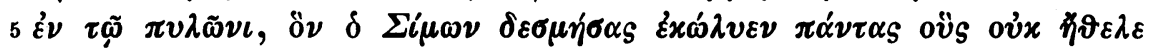

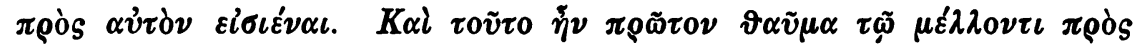

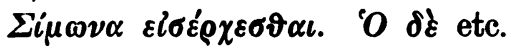

1) F. Hirsch, Byzantinische Studien, Leipzig 1876, p. 14 s. - C. de Boor, Zur Kenntnis der Weltchronik des Georgios Monachos, dans les Historische Untersuchungen Arnold Schaefer gewidmet, Bonn 1882, p. 276 ss.

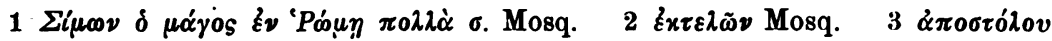

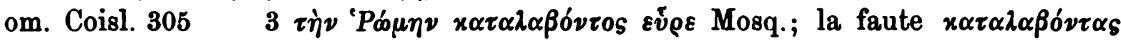

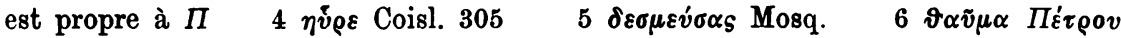

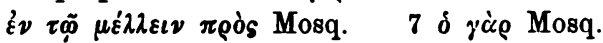


Le Patmiacus reproduit donc textuellement la même version que le Coislinianus 134, version que le Coisl. 305 présente avec quelques altérations. Les groupements de variantes sont à peu près les mêmes pour la suite du chapitre 121, ainsi que pour les chapitres 122 et 123. A la fin du chapitre 123, la version du Patmiacus est caractéristique, et elle concorde encore une fois avec celle du Coislinianus 134; il écrit

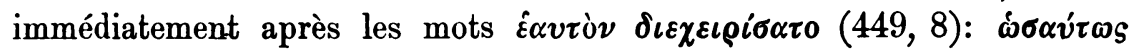

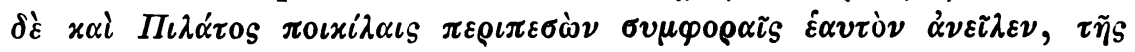

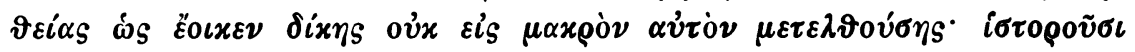

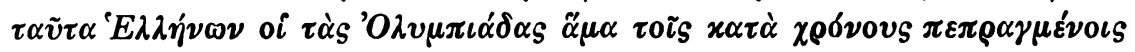

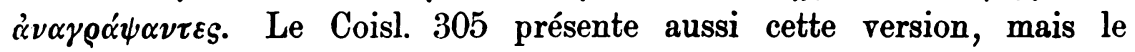
passage figure dans une suite de feuillets où l'écriture n'est pas de la première main.

Les règnes de Galba, d'Othon, de Vitellius et de Vespasien sont résumés en quelques lignes (chap. 124, 125, 126 et 127); nous ne noterons ici que les variantes des deux Coisliniani 134 et 305 .

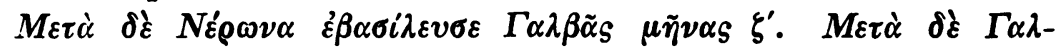

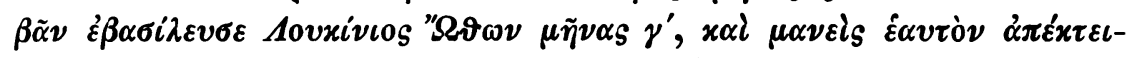

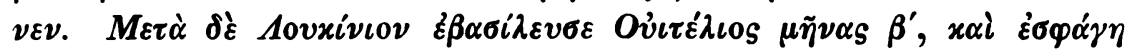

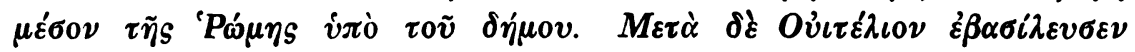

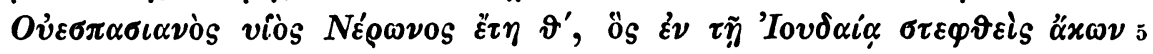

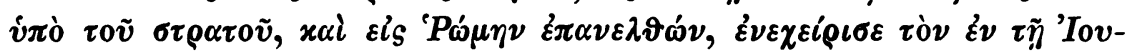

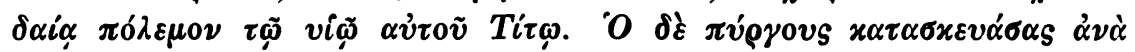

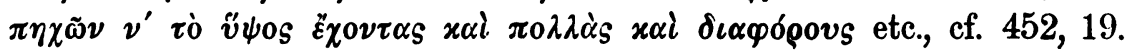

La longue narration de la prise de Jérusalem figure au complet dans le Patmiacus. Très souvent, le texte du Patmiacus s'écarte de celui du Mosquensis, pour présenter la leçon de Cedrenus. Malheureusement nous ne connaissons pas pour ce long passage la version des autres manuscrits. Il en est de même pour les chapitres $129\left(\tilde{5} 0 \check{5}, 48\right.$ : $\varepsilon \sigma \xi \beta^{\prime}$ $\Pi$ comme Cedrenus), $130,131,132$ et 133 . La fin du chapitre 134

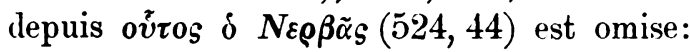

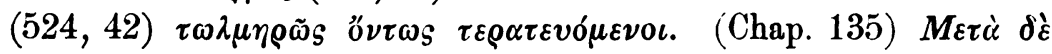

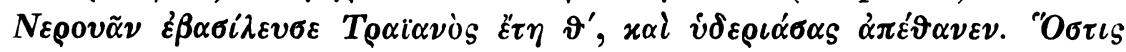

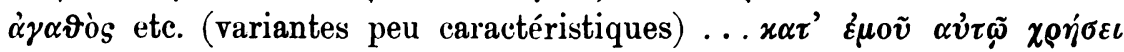

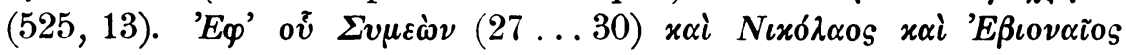

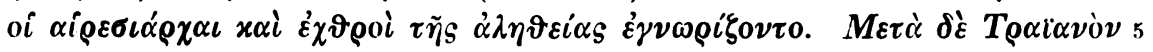

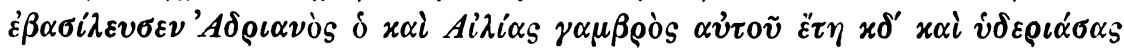
$\dot{\alpha} \boldsymbol{\pi} \varepsilon \boldsymbol{v} \boldsymbol{\alpha \nu \varepsilon \nu}$.

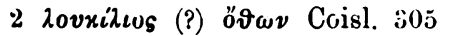

Coisl. 1345 ở
3 גouxiliuv Coisl. 305

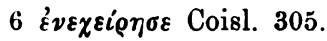

lovethlos sis

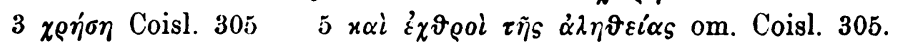


Pour tout ce passage, nous nous sommes assuré que le Coisl. 134 et $\Pi$ reproduisent un texte identique.

Les chapitres suivants jusqu'au règne de Didius occupent à peine une page du Patmiacus. Puis viennent les chapitres 142 et 143 , où un travail récent de $M$. Lauchert ${ }^{1}$ ) nous permet d'établir un rapprochement intéressant; voici le texte du Patmiacus, et, en note, les variantes des deux Coisliniani et du Strassburgensis; nous laissons de côté à partir d'ici les leçons du Mosquensis, dont la version est tout autre.

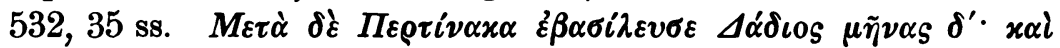

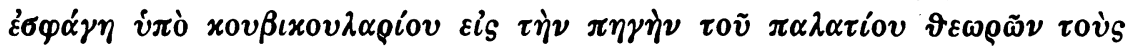

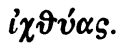

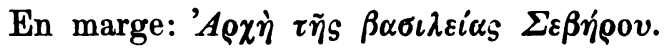

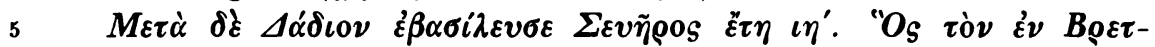

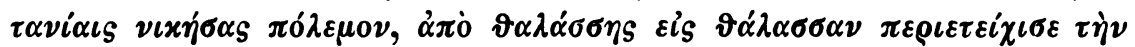

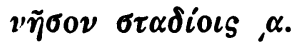

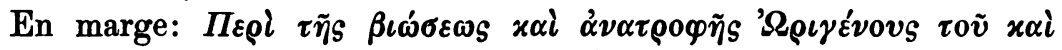
'Ad $\alpha \mu \alpha \nu \tau i o v$.

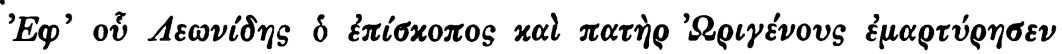

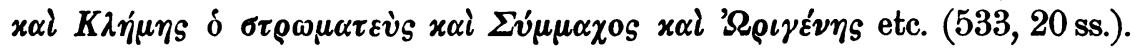

L'étroite parenté du Patmiacus avec le Coisl. 134 devient de plus en plus visible.

Le passage relatif à Origène est donné par $\Pi$ sans lacune, mais avec un bon nombre des variantes de Cedrenus; puis, les règnes des successeurs de Sévère (ch. 144-151) occupent à peine une page du manuscrit, c'est-à-dire la valeur de 25 lignes environ de l'édition de Migne. L'histoire du juif baptisé (ch. 151) est reproduite en entier. Les ch. 152, 153, 154 occupent à peine 6 lignes, par contre le chapitre sur la peste (155) est cité tout au long; nous pouvons désormais nous abstenir de faire remarquer que $\Pi$ a un bon nombre des leçons de Cedrenus. Après un résumé très court des règnes de Gallus et de ses successeurs jusqu'à Aurélien, le Patmiacus donne sans omission les

1) Zur Textïberlieferung der Chronik des Georgios Monachos, Byzantinische Zeitschr. 4 (1895) 497.

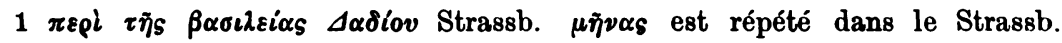

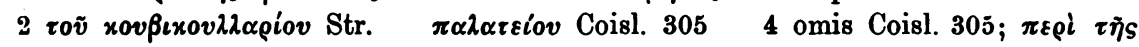

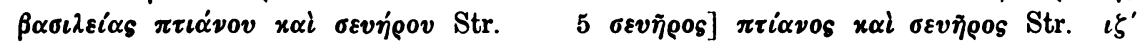

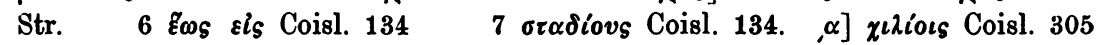

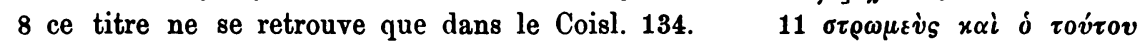

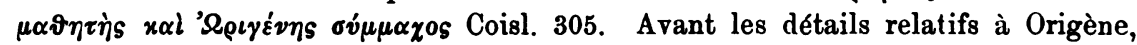

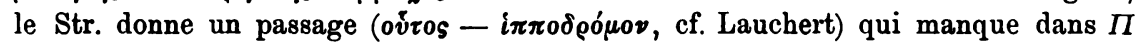
et dans les Coisliniani 
chapitres relatifs à Manès, Paul de Samosate, Apollinaire, Théodore de Mopsueste et Eutychès; extrêmement bref pour les règnes des empereurs depuis Tacite jusqu'à Dioclétien, il donne ensuite avec des variantes

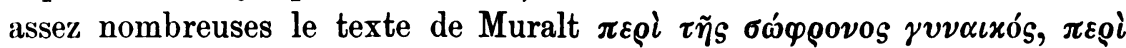

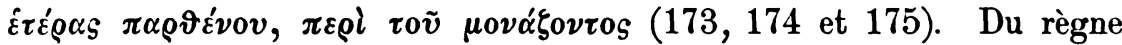
de Constance il a seulement, avec le titre, le premier alinéa $(573,6$ à 12); puis il continue:

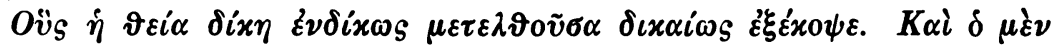

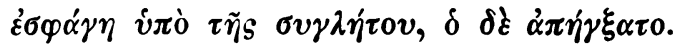

Le Coisl. 134 a le même texte. Après $\dot{\alpha} \pi \eta \dot{\gamma} \gamma \xi \alpha \tau o$, le Coisl. 305

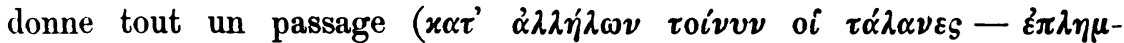
$\mu \varepsilon^{\prime} \lambda \eta \sigma \alpha \nu \delta \iota \alpha \delta \iota \delta \rho \alpha \sigma \delta \varepsilon \iota \nu$ $\left.\tau \iota \mu \omega \rho i \alpha \nu\right)$ qui manque dans $\Pi$ et dans le Coisl. 134.

Pour le passage suivant, nous reproduirons le texte de $\Pi$ avec les variantes des deux Coisliniani; on verra qu'il s'écarte à la fois du Mosquensis de Muralt, et de la version commune au Strassburgensis et à l'Ambrosianus (cf. Lauchert, étude citée, p. 498 s.):

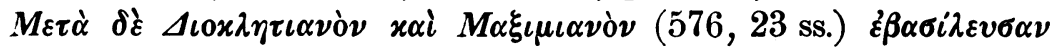

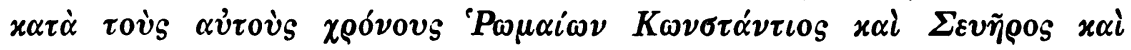

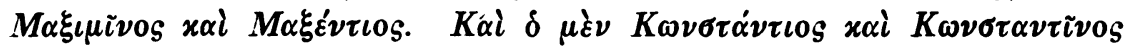

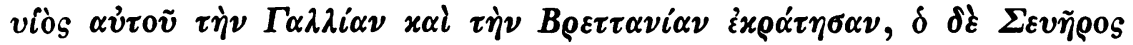

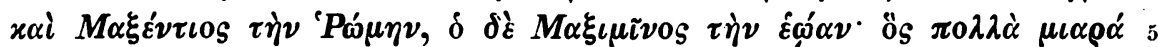

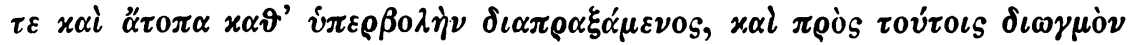

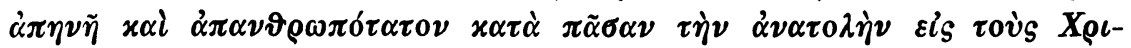

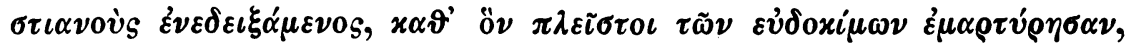

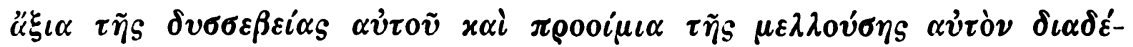

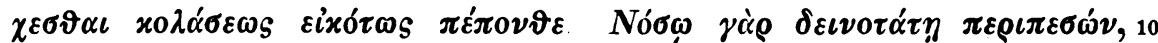

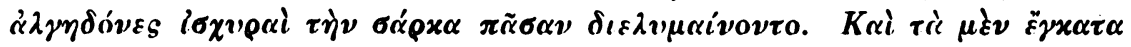

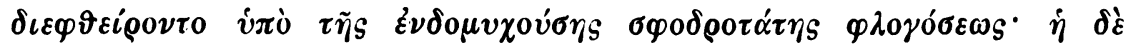

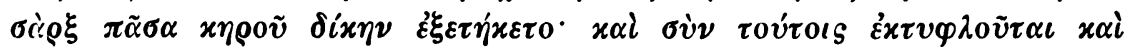

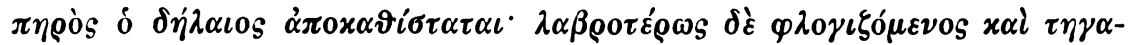

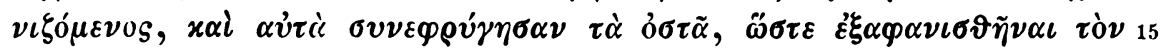

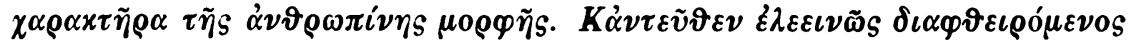

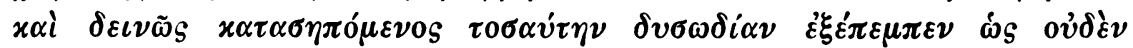

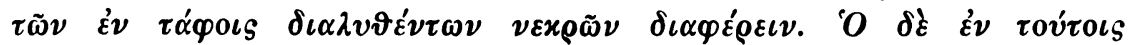

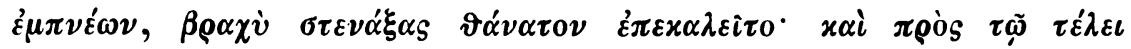

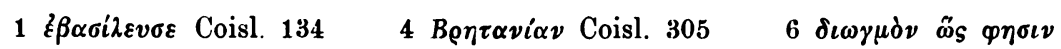

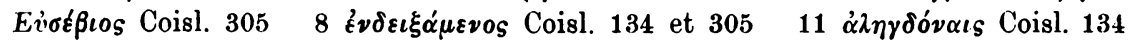

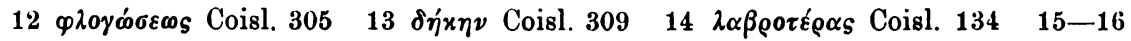

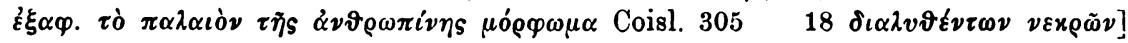
$\chi \alpha \tau \alpha \varphi \vartheta \varepsilon \iota \rho \circ \varepsilon \dot{\varepsilon} \omega \nu$ Coisl. 305 


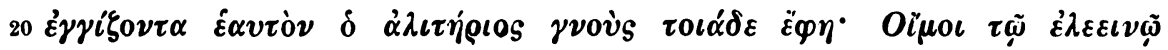

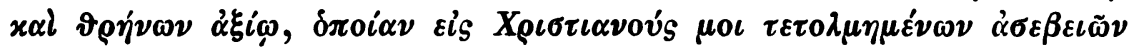

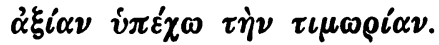

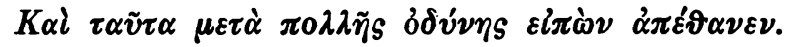

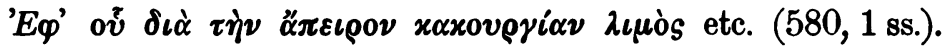

Nous avons hâte d'arriver à des passages où des renseignements plus complets permettront des conclusions plus instructives. Signalons rapidement les lacunes importantes du Patmiacus dans les chapitres qui suivent. La fin du règne de Constance est singulièrement écourtée.

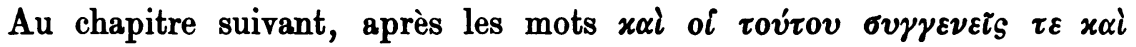

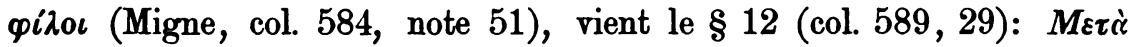

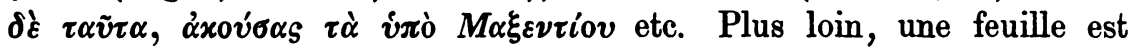

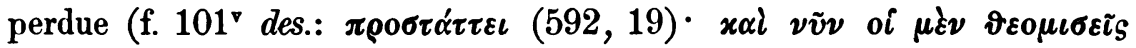

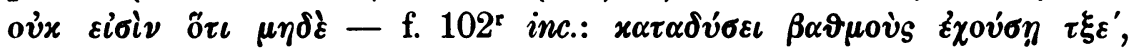
$593,34)$. On ne voit donc pas si le Patmiacus avait placé, dans ce passage, une séparation entre les livres III et IV. Tandis que le Coisl. 305 ne marque aucune division, le Cois. 134 a en grands ca-

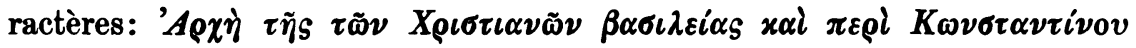

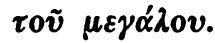

Pour le commencement du livre IV jusqu'à la page $451,1.16$ de son édition (col. 676 Migne), Muralt a donné les variantes de l'Ambrosianus, des deux Vindobonenses hist. 40 et $2\left(V^{1}\right.$ et $\left.V^{2}\right)$ et des Monacenses Gr. 414 et $139\left(\mathbf{M}^{1}\right.$ et $\left.\mathbf{M}^{2}\right)$. La plupart des ces variantes se retrouvent dans le Patmiacus, mais de tous ces manuscrits c'est $V^{1}$ qui, avec le Coisl. 134, est le plus voisin de $\Pi$. Voici un exemple de leurs concordances.

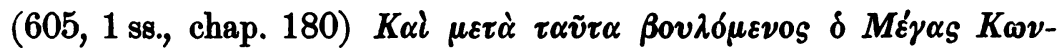

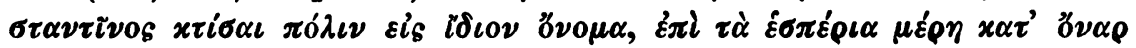

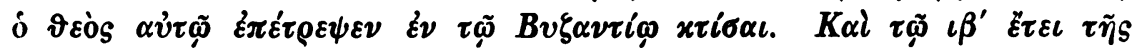

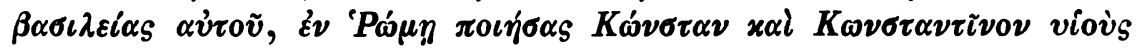

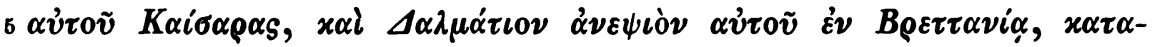

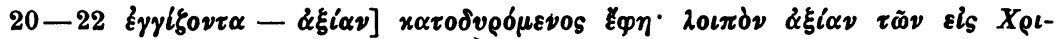

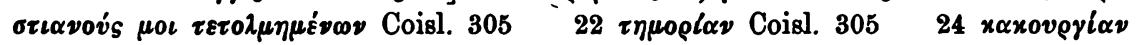
$\alpha \tilde{\tau} \tau \tilde{v}$ Coisl. 305

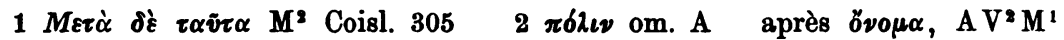
et $M^{2}$ ont, comme le Mosq., un passage que $\Pi V^{1}$ et les Coisl. remplacent par

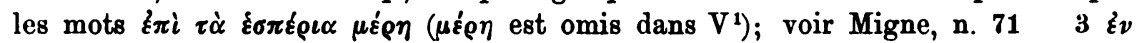

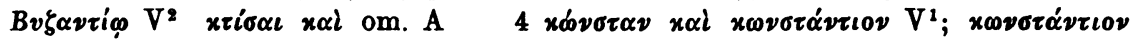

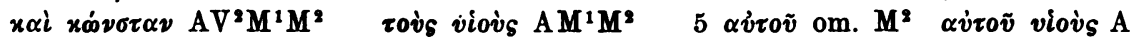

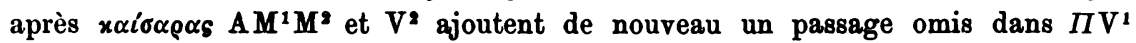

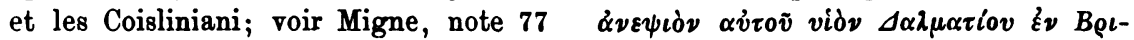
$\tau \alpha \nu i \alpha x_{i} x \alpha i \quad x \alpha \tau \alpha \lambda \varepsilon i \psi \alpha_{s}$ Coisl. 305 


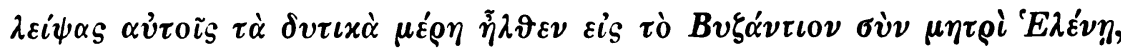

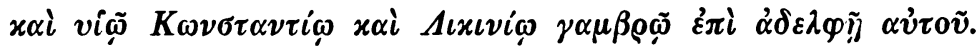

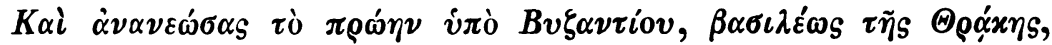

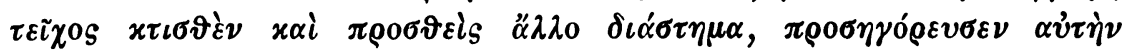

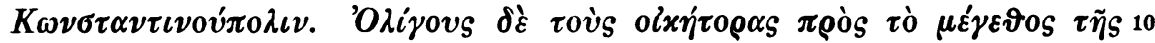

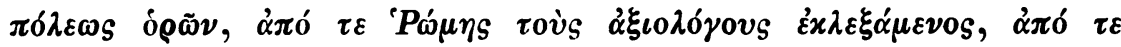

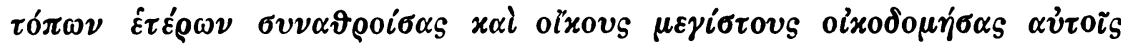

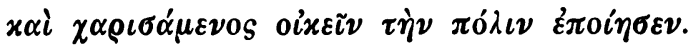

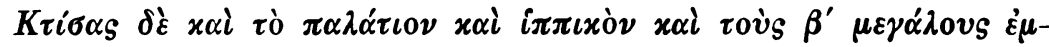

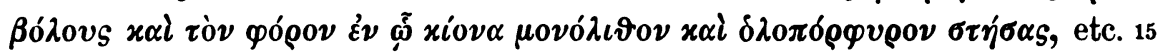
$(605,28)$.

De tous les manuscrits dont Muralt donne les variantes, $V^{\mathbf{1}}$ est donc le seul qui présente, à peu de chose près, le même texte que $\Pi$ et Coisl. 134. Comme on le verra par la suite, cette rencontre n'est pas une pure coïncidence.

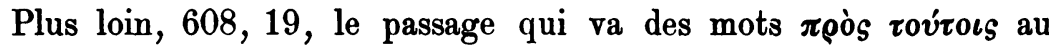

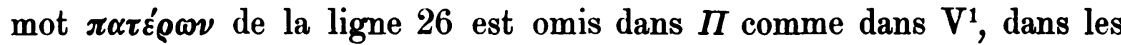
deux Monacenses et dans les deux Coisliniani.

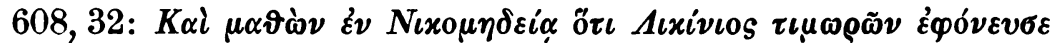

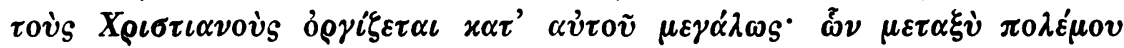

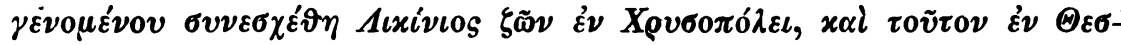

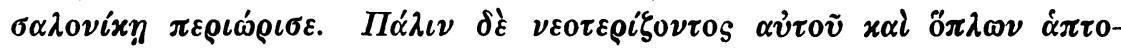

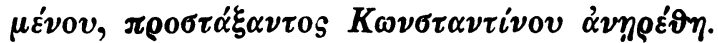

' $H$ d' $\tilde{\varepsilon} \mu \alpha x \alpha \rho i \alpha$ etc. $(609,15$ ss.).

Cette version s'écarte beaucoup de celle du Mosquensis, du Coislinianus 305 et de l'Ambrosianus, très peu de celle des Monacenses et de $\mathrm{V}^{2}$, absolument pas de celle du Coisl. 134. Le peu de précision

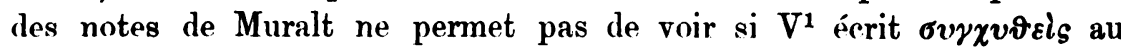

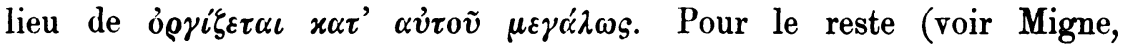
notes 27 et 35), il présente absolument le même texte que $\Pi$, sauf

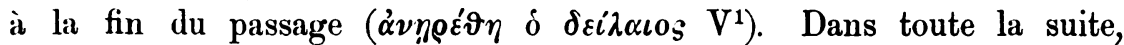
les rapports entre les deux manuscrits restent des plus étroits. L'un

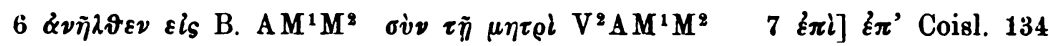

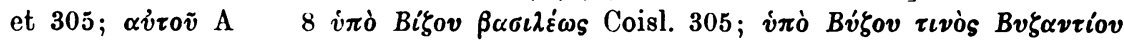

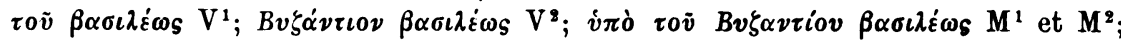

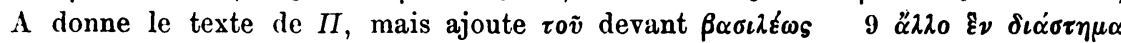

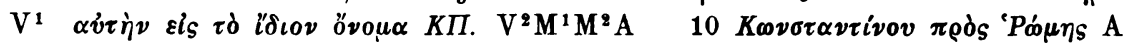
ỏhiyovs jusqu'à 'Ṕóns 605, 27 om. $\mathrm{M}^{1} \tau \tilde{\eta} s$ om. $\mathrm{V}^{1} \mathrm{~V}^{2} \mathrm{M}^{1} \mathrm{M}^{2} \mathrm{~A} \quad 11 \delta \rho \tilde{\nu} \nu, \tau i$

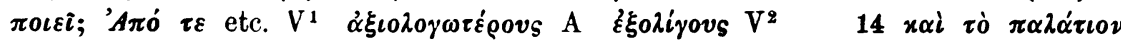

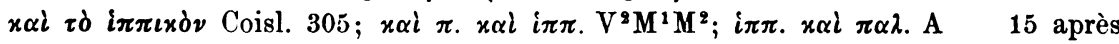
$\dot{\varepsilon} \nu \stackrel{⿱ 亠}{\omega}$ les manuscrits ajoutent une ligne que $\Pi$, les deux Cisl. et $V^{1} V^{2}$ omettento 
et l'autre omettent le récit de l'invention de la croix et reprennent

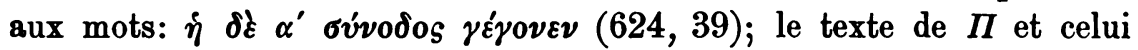
de $\mathrm{V}^{1}$ coïncident ici comme partout ailleurs. ${ }^{1}$ )

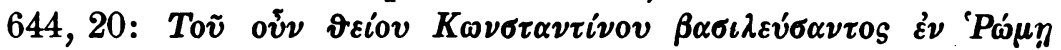

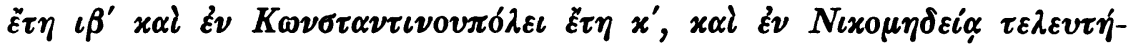

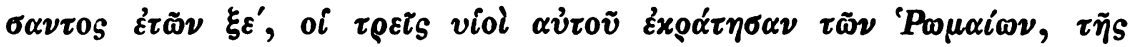

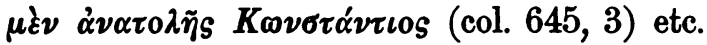

Le texte est le même dans les Coisl. 134 et $\left.305^{2}\right) ; V^{1}$ ne s'en écarte que dans des variantes insignifiantes ( $\dot{\varepsilon} \tau \tilde{\omega} \nu$ omis à la l. 4).

$661,1-664,15]$ om. $\Pi$. Pour tout ce passage, $V^{1}$ ne figure pas une seule fois dans l'appareil critique de Muralt; ce manuscrit a donc ici la même omission que $\Pi$. L'un et l'autre présentent une lacune identique au début du règne de Julien $(664,18$ Kaĩ $\alpha \rho-$ ibid. 26 $\dot{\alpha} \pi \varepsilon \pi \lambda v^{2} \alpha \tau$ ). A partir de la page 451, 16 (col. 676), Muralt ne donne plus les variantes des Vindobonenses.

Le Patmiacus omet dans la suite, au chapitre 192, le $\S 6$; col.

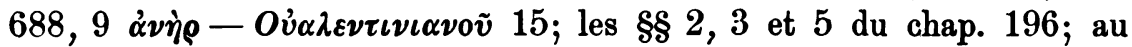
chap. 198, la première moitié du $\S 11$, et le $\S 12$ en entier; au $\S 13$, il donne un texte différent de celui de Muralt; il omet encore les deux premiers $\S \S d u$ chap. 200; puis, dans le même chapitre, les $\S \S 5,6$ et 7 ; chap. 205 , le $\S 7 ; 206, \S 1,10$ et $11 ; 207, \S 5$, r0 et $11 ; 208, \S 8,9$ et 10 .

Nous voici au règne de Léon $(756,23)$, où un rapprochement des différentes versions sera de la plus grande utilité.

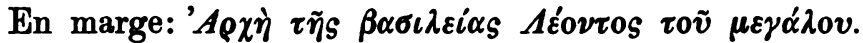

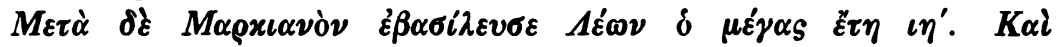

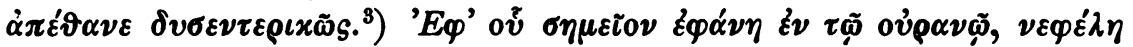

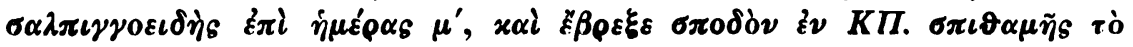

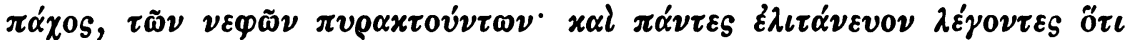

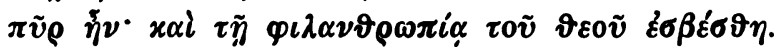

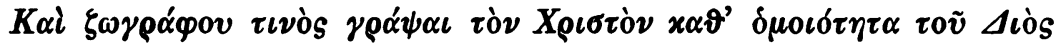

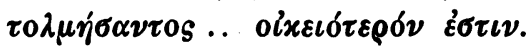

1) Nous n'avons collationné le Coisl. 134 que pour les passages de $\Pi$ que nous reproduisons; il est vraisemblable qu'ailleurs aussi ils sont d'accord entre eux et avec le Coisl. 310 .

2) Les variantes du Coisl. 305 que Muralt cite dans son introduction (Migne, p. 19) ne se retrouvent naturellement pas dans le Patmiacus. M. de Boor (étude citée, p. 282) a fait remarquer que le Coisl. 305 modifie sonvent avec beaucoup d'arbitraire le texte de son archétype. A partir d'ici, nous pouvons laisser de côté les leçons de ce manuscrit.

3) „Im Vaticanus 153 ist die Formel durch Umstellung der Worte gestört" (de Boor, p. .278). 


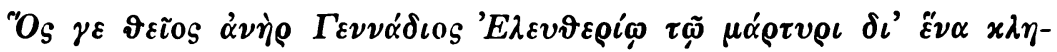

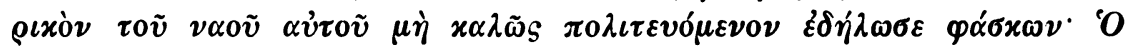

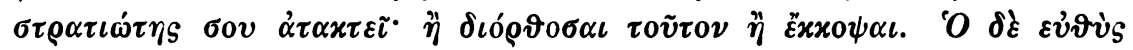

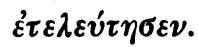

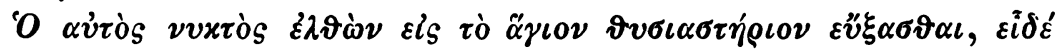

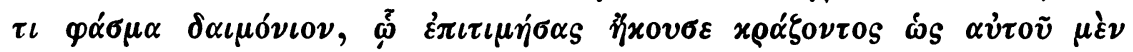

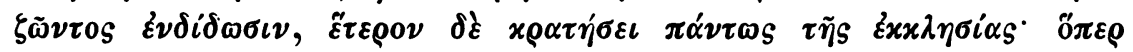

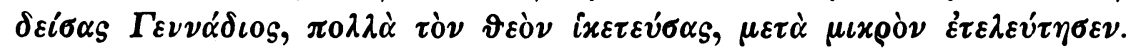

Ici encore, le Patmiacus reproduit, à part quelques fautes, la version des Coisl. 310 et 134 (voir, outre Muralt, Lauchert, p. 501, et de Boor, p. 278 ss.).

Après $\dot{\varepsilon} \tau \varepsilon \lambda \varepsilon \dot{v} \tau \eta \sigma \varepsilon$, le Mosquensis donne des détails (\$§ 6, 7, 8 et 9), qui manquent dans le Patmiacus comme dans le Strassburgensis et les Coisliniani 310 et 134 (nous laissons de côté, comme nous l'avons annoncé plus haut, le Coisl. 305).

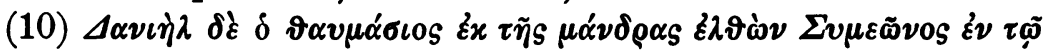

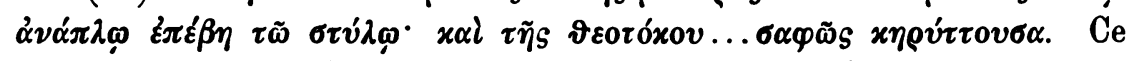
passage de $\Pi$ cö̈ncide à peu près avec le texte du Strassb. (Lauchert, p. 202) et complètement avec celui des Coisl. Ceux-ei ont deux

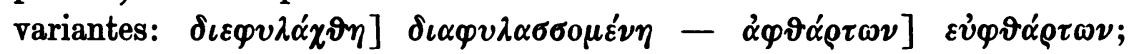
elles se retrouvent dans le Patmiacus. Enfin l'interpolation du Strassb.:

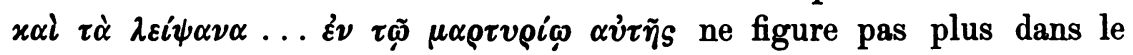
Patm. que dans les Coisl.

Le Patm. omet le $\S 12$, puis vient la courte notice sur Léon II:

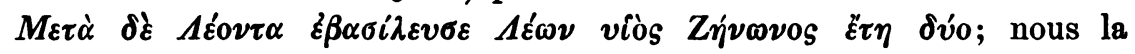
reproduisons en entier, parce qu'elle permet de voir que $\Pi$ n'a pas les leçons spéciales au Vaticanus 153, qui est lui aussi un représentant de la classe du Coisl. 310 (voir de Boor, p. 284). Puis vient

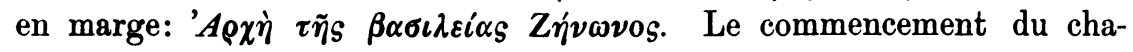
pitre 221 est textuellement le même que dans les Coisliniani: $Z \eta \dot{\nu \omega \omega} \delta$

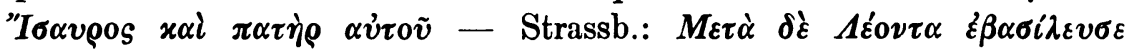

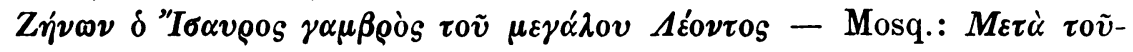

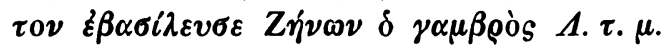

Les $\S \S 4$ et 5 sont omis dans le Patmiacus comme dans tous les autres manuscrits, excepté le Mosquensis.

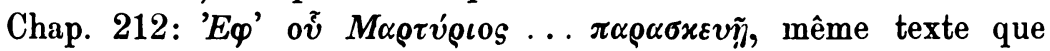
le Str. (Lauchert, p. 504), sauf les variantes des Coisl., qui se retrou-

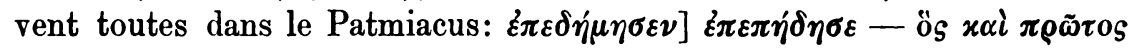
$\varepsilon \pi \varepsilon v{ }^{\prime} \eta \sigma \varepsilon$ (tandis que le Vatic. 154 se rapproche du Str., voir Laauchert,

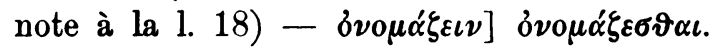

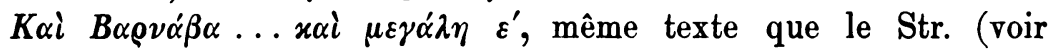


Lauchert, p. 504-505) avec quelques variantes peu importantes, variantes que nous avons retrouvées dans le Coisl. 134.

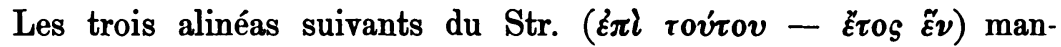
quent dans le Patmiacus ainsi que dans les Coisliniani; ce passage n'est, comme M. Lauchert le fait observer, qu'une note marginale, que le copiste du Str. a eu le tort d'introduire dans le texte.

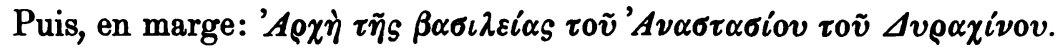

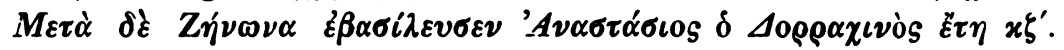

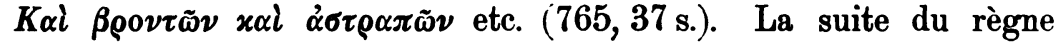
d'Anastase me paraît être la même que dans les Coisliniani 310 et 134. Notons que $\Pi$ ici encore suit les Coisl. plutôt que le Vaticanus 153,

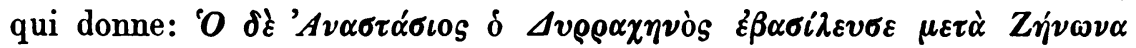

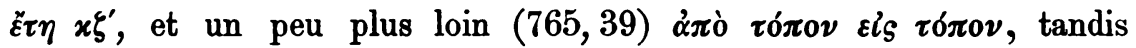

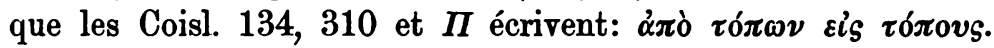

Pour les chapitres suivants, une description très sommaire suffira.

Le Patmiacus omet, au chap. 215 , les $\$ \$ 14$ à 17 ; au chap. 216, la fin du $\S 1$, et les $\S \S 2$ à 6 , la fin du $\S 10$, et les $\S \S 11$ à 15 ; au chap. 217, les $\$ 82$ et 3 .

Pour une partie considérable du chapitre 220, la disparition d'une feuille du Mosquensis a forcé Muralt à reproduire le texte d'un autre manuscrit; il a adopté celui du Coisl. 305. Le Patmiacus présente pour tout ce passage des variantes assez nombreuses; il omet col. 797, 33,

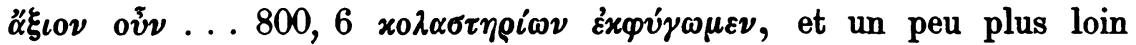
$800,41 \tau \eta \tilde{g}$ $\tau \alpha \lambda \alpha \iota \pi \omega \rho i \alpha s . .47$ oĩ $\gamma \varepsilon$.

La fin du même chapitre 220 ( $\$ \S 9$ à 15) manque également dans $\Pi$. Le règne de Tibère (chap. 223) esst mentionné en quelques

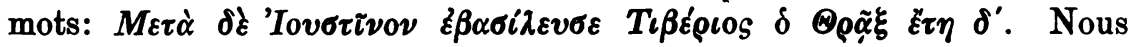
avons retrouvé ces deux particularités dans le Coisl. 134.

Chap. 224, le texte du Patmiacus est plus court que celui de Muralt, et présente des variantes considérables; puis vient, avec très peu de lacunes, l'histoire des sirènes, des crocodiles et des hydres (ch. 225). Un certain nombre des variantes de $\Pi$ se retrouvent dans Cedrenus ou dans les Monacenses, dont Muralt donne ici les leçons.

A partir de la col. 825 jusqu'à la colonne 836 , nous retrouvons dans l'apparat critique de Muralt les variantes du Vindobonensis $40^{1}$ ); elles sont absolument les mêmes que celles du Patmiacus.

1) Quand les annotations de Muralt nous paraissaient peu claires, nous avons recouru à sa source, Tafel, Theophanis Chronographia, Probe einer newen kritischexegetischen Ausgabe, dans les Sitzungsber. der Wiener Akademie, 1853, t. IX, p. 44 ss., où Tafel reproduit un passage de Georges le Moine d'après le Vindobonensis 40. 
Un exemple de ces concordances achèvera de démontrer que les trois manuscrits $\Pi, \mathrm{V}^{1}$, Coisl. 134 présentent une seule et même version de la chronique de Georges le Moine.

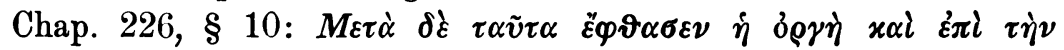

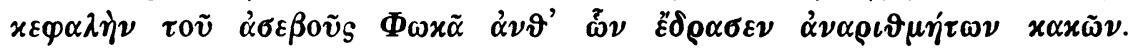

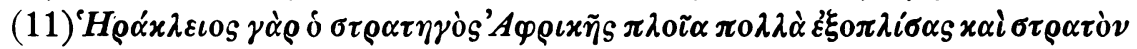

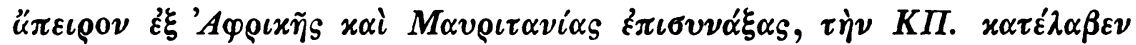

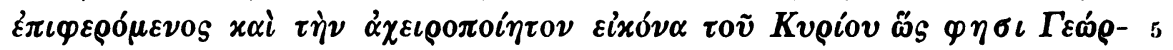

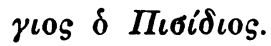

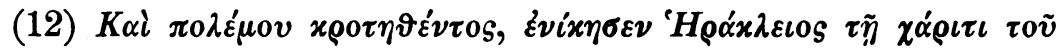

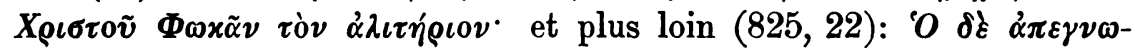

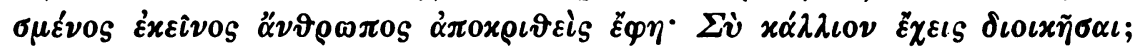

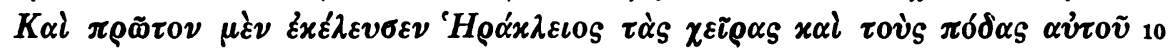

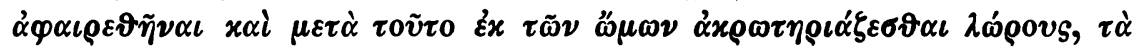

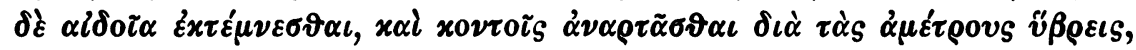

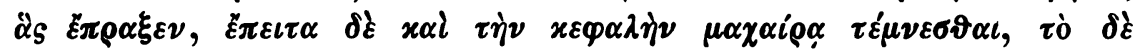

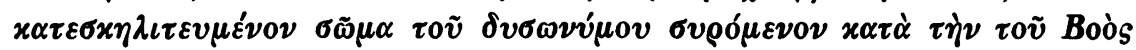

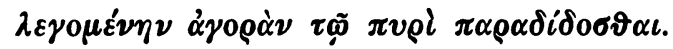

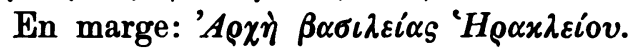

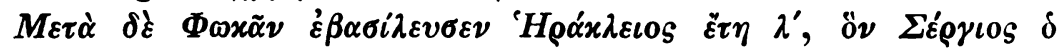

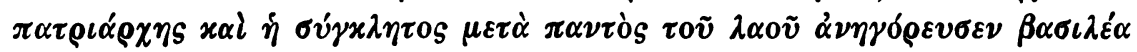

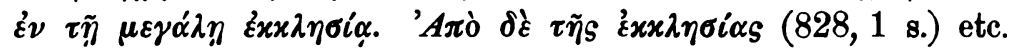

Il est inutile de continuer à noter les variantes des Mosq. et Mon.: le lecteur les verra très aisément en rapprochant ce texte de celui de Muralt, dont les Mon. different peu ici; quant à $V^{1}$ et Coisl. 134, ils ont identiquement le même texte que $\Pi$, à part quelques fautes, variantes ou additions peu importantes de $\mathrm{V}^{1}$ (par ex. 1. 4 Mavo.

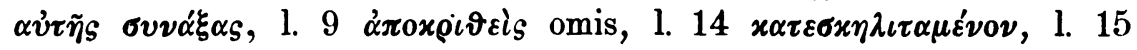
$\pi \alpha \rho \alpha \delta \varepsilon \delta \delta ́ \sigma \vartheta \alpha \iota)$.

Sautons une colonne, où les deux manuscrits $\Pi$ et $\mathrm{V}^{1}$ (Coisl. 134 sans doute aussi) présentent toujours le même texte, restant d'accord dans tous les détails, même dans les plus petites fautes. Pour achever la démonstration, voici le texte commun aux trois manuscrits dans un passage où la version de Muralt est très différente $(829,5 \mathrm{ss}$.):

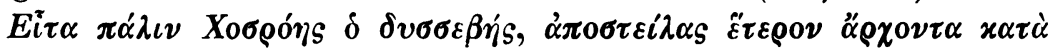

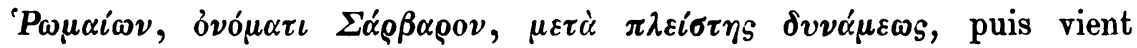
avec quelques variantes peu importantes le texte du Vind. cité à la note 85 de Migne; ensuite:

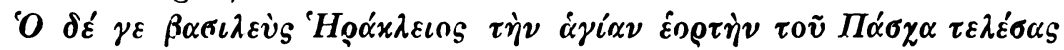

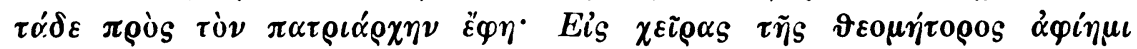

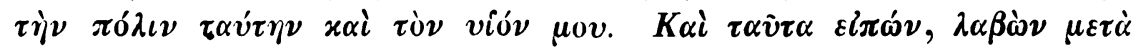




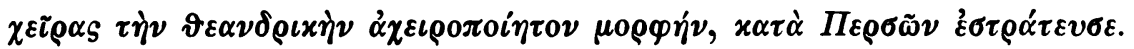

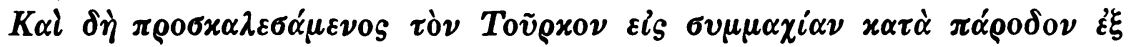

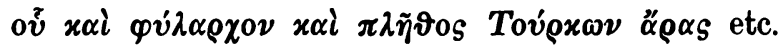

C'est là à très peu de chose près le texte de $\mathrm{V}^{1}$, comme on peut le voir aux notes que Migne' a placées sous la colonne 829: comme toujours, $\Pi$ et le Coisl. 134 restent d'accord là où $V^{1}$ présente une variante.

$\S 13$ om. dans $\Pi$ et $\mathrm{V}^{1}$; au $\S 14, \Pi$ a le texte de $\mathrm{V}^{1}$, ainsi que pour toute la fin du chapitre; les différences sont très légères; il en est de même dans le court chapitre 228. Après ce chapitre, s'arrête la collation du Vindobonensis.

Chaque fois qu'on connait le texte de $\mathrm{V}^{\mathbf{1}}$ en même temps que celui de $\Pi$, on les voit différer en si peu de chose, qu'il ne peut rester aucun doute; le Vindobonensis suit le Patmiacus presque d'aussi près que celui-ci suit le Coislinianus 134 et sans doute aussi le Coisl. 310. Les quatre manuscrits forment un groupe à part, et c'est ce groupe qui reproduit le plus fidèlement l'oeurre de Georges le Moine, tandis que le Vaticanus 153 et le Coislinianus 305 présentent la version de ce même groupe, mais chacun avec des altérations du texte primitif qui leur sont spéciales.

Dans la suite, le Patmiacus omet encore entre autres le chap. 233; au chap. 234 , le $\S 1$ sauf la première phrase, le $\$ 3$ et le commencement du $\S 4$; au chap. 239 , le $\S 6$, les $\S \S 12$ à 19 ; au chap. 240 , les $\S \S 1$ et 2 . Puis il y a encore un bon nombre de passages omis ou écourtés. Mais nous avons indiqué assez de lacunes pour que le contrôle de nos conclusions puisse se faire sur une portion considérable du texte de la chronique.

Nous avons, pour les dernières feuilles du Patmiacus, des collations très nombreuses: à la fois, outre celle du Mosquensis, celles des Coisl., du Par. 1705, du Strassburgensis, et, pour le début du chapitre 262, des Monacenses. Voici quelques spécimens du texte du Patmiacus. Afin d'éviter une surcharge de notes inutile ici, nous donnerons seulement les variantes des Coisliniani 310 et 134.

Ch. 262; titre omis.

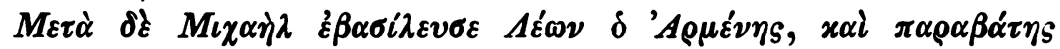

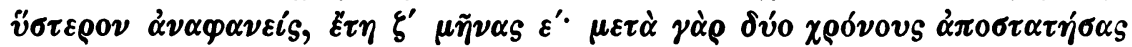

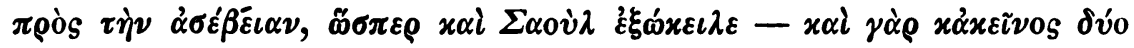

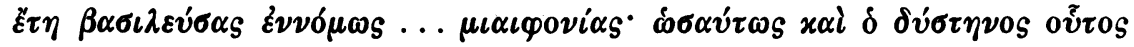

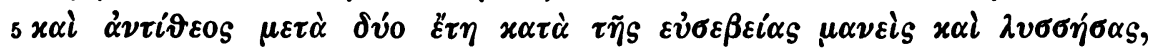

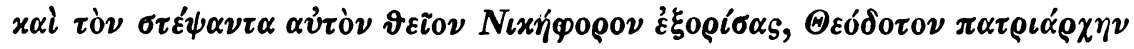

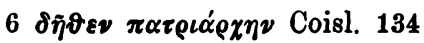




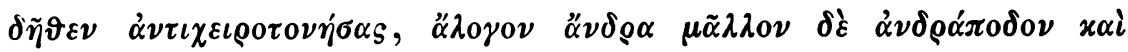

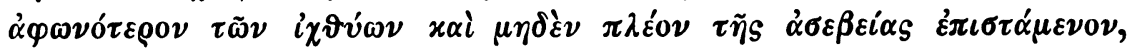

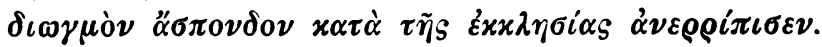

Seuls les Coisliniani 134 et 310 ont le même texte que le Patmiacus, tandis que les Monacenses s'accordent plutôt avec le Strassburgensis, et le Parisinus avec le Mosquensis.

Le Patmiacus omet les $\S \S 3$ et 4 comme les Coisl., et les groupements des manuscrits restent les mêmes pour la suite du chapitre. A la fin du $\S 5$, il y a des variantes intéressantes; le texte du Strassbur-

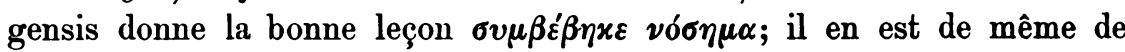
$\mathrm{V}^{1}$, d'après une copie que nous devons à l'obligeance de $\mathbf{M}$. A. Göldlin von Tiefenau. La perte d'un feuillet a fait disparaître tout ce passage du Coisl. 134, et le texte du Coisl. 305 s'arrête beaucoup plus haut; quant au Coisl. 310, il donnerait vóou $\mu \alpha$ ? d'après une note de Muralt

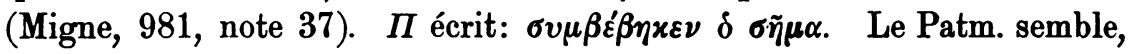
là où nous connaissons les leçons du Coisl. 310, plus près de ce manuscrit que de tout autre. Ici tous deux ont altéré la bonne leçon. Remonteraient-ils à un même archétype où le mot était peu lisible? N'ayant pas pu consulter le Coisl. 310, et les collations de ce codex n'étant publiées qu'en très petit nombre, nous devons laisser à l'éditeur de Georges le Moine le soin de résoudre cette question, à notre avis très importante.

La perte de deux feuillets a fait disparaitre du Patmiacus une partie assez considérable du même chapitre 262, depuis les mots

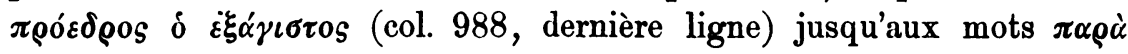

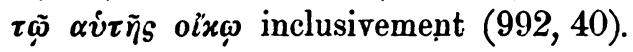

Nous donnerons un dernier extrait du texte du Patmiacus, extrait où il s'écarte une ou deux fois du Coislinianus 310.

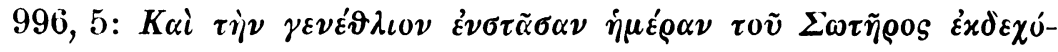

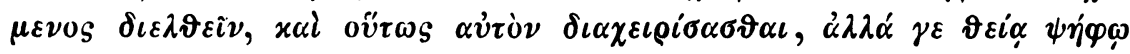

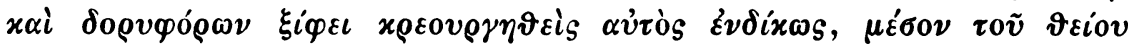

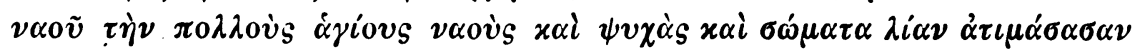

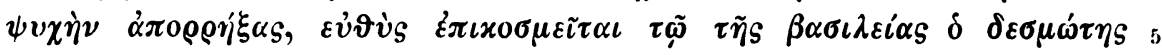

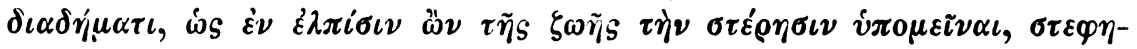

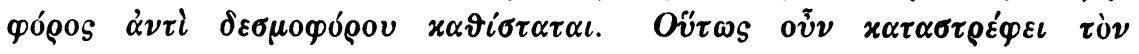

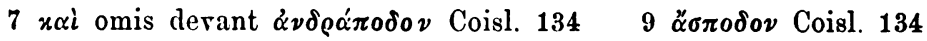

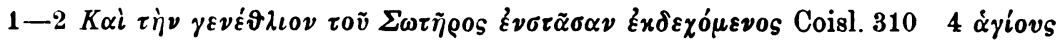

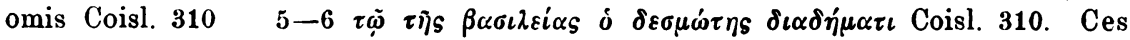
trois variantes sont exprantúés ì la note 55, col. 996, de Muralt. Cette note attribue le même texte aux Coisl. 310 et 134 ; ces variantes cependant ne se retrourent pas dans le Coisl. 134

Byzant. Zeitschrift VIJ 2.

20 


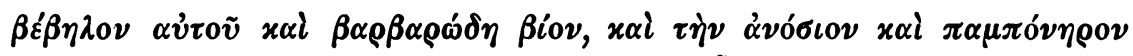

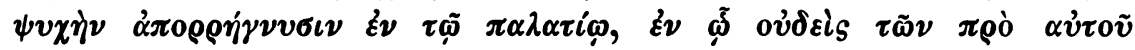

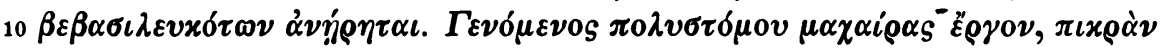

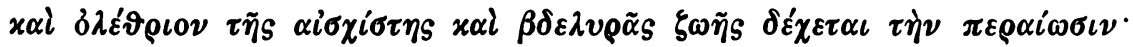

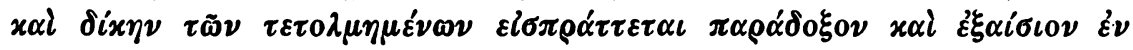

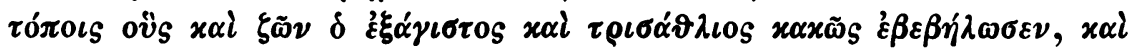

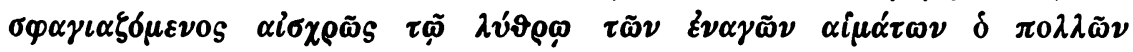

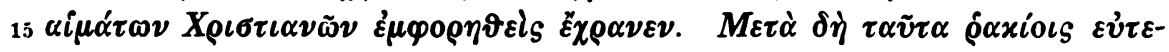

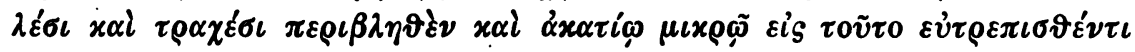

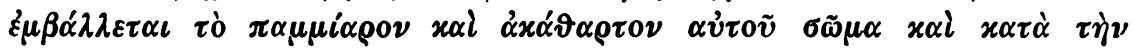

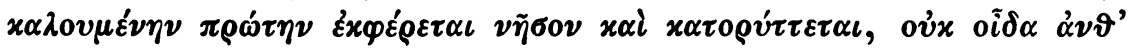

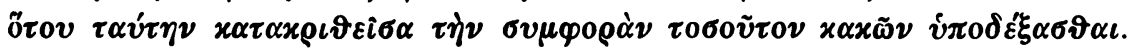

Le manuscrit s'arrête au bas du feuillet $295^{\circ}$, avant la fin du

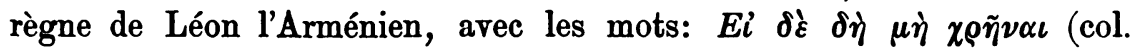
$1000,1.10)$.

La conclusion de cette série de rapprochements est déjà tout indiquée. Les manuscrits connus de la chronique de Georges le Moine paraissent se ranger dans trois grandes catégories: celle du Mosquensis, celle du Strassburgensis et celle du Coislinianus 310. C'est à la dernière que le Patmiacus appartient, en même temps que les Coisliniani 134 et 305, le Vindobonensis 40 et le Vaticanus 153. Le Patmiacus ne paraît pas dériver d'un de ces manuscrits. Il ne reproduit pas les quelques variantes qui sont spéciales au Vindobonensis, pas plus que les particularités de rédaction du Vaticanus ou du Coislinianus 305. Laissant chacun de ces deux derniers manuscrits isolé, il ajoute encore à la vraisemblence des conclusions de $\mathbf{M}$. de Boor, pour qui les leçons spéciales à l'un et à l'autre proviennent de remaniements arbitraires. Le Patmiacus reproduit avec assez d'exactitude la version de l'archétype commun à tout le groupe. Faut-il, dans ce groupe, établir une subdivision, dans laquelle on ferait entrer le Patmiacus avec les Coisliniani 310 et 134 ? Nous connaissons trop rarement le texte du Coisl. 310 en même temps que celui des autres manuscrits, surtout du Vindobonensis, pour être à même de le dire. Si la question mérite d'être étudiée, elle pourra l'être avec beaucoup plus de chances de succès par le futur éditeur de la chronique de Georges le Moine.

Gand.

J. Bidez.

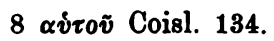

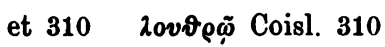
Coisl. 134

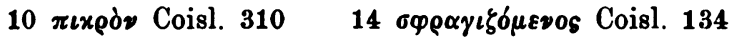

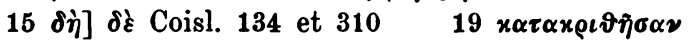

